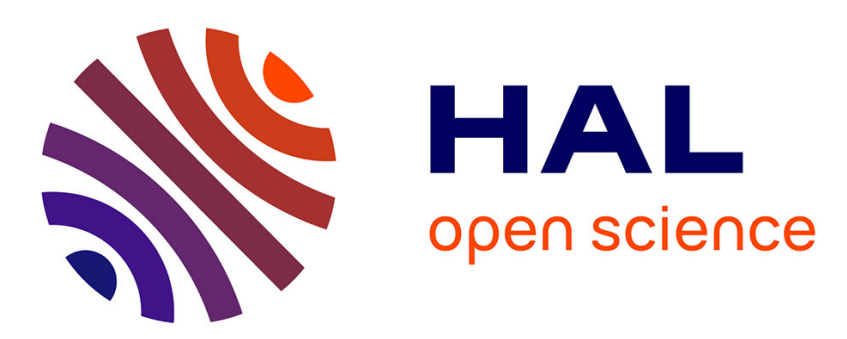

\title{
Elliptically polarized light for depth resolved optical imaging
}

Anabela Da Silva, Carole Deumie, Ivo Vanzetta

\section{To cite this version:}

Anabela Da Silva, Carole Deumie, Ivo Vanzetta. Elliptically polarized light for depth resolved optical imaging. Biomedical optics express, 2012, 3 (11), pp.2907-2915. 10.1364/BOE.3.002907 . hal01772629

\section{HAL Id: hal-01772629 \\ https://hal-amu.archives-ouvertes.fr/hal-01772629}

Submitted on 20 Apr 2018

HAL is a multi-disciplinary open access archive for the deposit and dissemination of scientific research documents, whether they are published or not. The documents may come from teaching and research institutions in France or abroad, or from public or private research centers.
L'archive ouverte pluridisciplinaire HAL, est destinée au dépôt et à la diffusion de documents scientifiques de niveau recherche, publiés ou non, émanant des établissements d'enseignement et de recherche français ou étrangers, des laboratoires publics ou privés. 


\title{
Elliptically polarized light for depth resolved optical imaging
}

\author{
Anabela Da Silva, ${ }^{, 1}$ Carole Deumié, ${ }^{1}$ and Ivo Vanzetta ${ }^{2}$ \\ ${ }^{1}$ Institut Fresnel, CNRS UMR 7249, Aix-Marseille Université, Ecole Centrale Marseille, Campus de St Jérôme, \\ 13013 Marseille, France \\ ${ }^{2}$ Institut des Neurosciences de la Timone, CNRS UMR 7289, Aix-Marseille Université, \\ Campus Santé Timone 13385 Marseille Cedex 05, France \\ *anabela.dasilva@fresnel.fr
}

\begin{abstract}
It is shown that using elliptically polarized light permits selecting well-defined subsurface volumes in a turbid medium. This suggests the possibility of probing biological tissues at specific depths. First, we present the method and preliminary results obtained on an Intralipid phantom. We next report on the method's performance on a biological phantom (chicken breast) and, finally, on the exposed cortex of an anesthetized rat.
\end{abstract}

(C) 2012 Optical Society of America

OCIS codes: (170.3660) Light propagation in tissues; (170.7050) Turbid media; (170.3880) Medical and biological imaging; (110.5405) Polarimetric imaging.

\section{References and links}

1. A. Grinvald, E. Lieke, R. D. Frostig, C. D. Gilbert, and T. N. Wiesel, "Functional architecture of cortex revealed by optical imaging of intrinsic signals," Nature 324(6095), 361-364 (1986).

2. A. Grinvald, T. Bonhoeffer, I. Vanzetta, A. Pollack, E. Aloni, R. Ofri, and D. Nelson, "High-resolution functional optical imaging: from the neocortex to the eye," Ophthalmol. Clin. North Am. 17(1), 53-67 (2004).

3. B. S. Sorg, B. J. Moeller, O. Donovan, Y. T. Cao, and M. W. Dewhirst, "Hyperspectral imaging of hemoglobin saturation in tumor microvasculature and tumor hypoxia development," J. Biomed. Opt. 10(4), 044004 (2005).

4. W. Steller, J. Einenkel, L. C. Horn, U. D. Braumann, H. Binder, R. Salzer, and C. Krafft, "Delimitation of squamous cell cervical carcinoma using infrared microspectroscopic imaging," Anal. Bioanal. Chem. 384(1), $145-154$ (2006).

5. V. V. Tuchin, Tissue Optics (SPIE Press, Bellingham, WA, USA, 2000).

6. F. C. MacKintosh, J. X. Zhu, D. J. Pine, and D. A. Weitz, "Polarization memory of multiply scattered light," Phys. Rev. B Condens. Matter 40(13), 9342-9345 (1989).

7. D. Bicout and C. Brosseau, "Multiply scattered waves through a spatially random medium: entropy production and depolarization," J. Phys. I 2(11), 2047-2063 (1992).

8. D. Bicout, C. Brosseau, A. S. Martinez, and J. M. Schmitt, "Depolarization of multiply scattered waves by spherical diffusers: Influence of the size parameter," Phys. Rev. E Stat. Phys. Plasmas Fluids Relat. Interdiscip. Topics 49(2), 1767-1770 (1994).

9. K. M. Yoo and R. R. Alfano, "Time resolved depolarization of multiple backscattered light from random media," Phys. Lett. A 142(8-9), 531-536 (1989).

10. R. R. Anderson, "Polarized light examination and photography of the skin," Arch. Dermatol. 127(7), 1000-1005 (1991).

11. S. L. Jacques, J. C. Ramella-Roman, and K. Lee, “Imaging skin pathology with polarized light,” J. Biomed. Opt. 7(3), 329-340 (2002).

12. S. G. Demos and R. R. Alfano, "Optical polarization imaging," Appl. Opt. 36(1), 150-155 (1997).

13. A. P. Sviridov, V. Chernomordik, M. Hassan, A. C. Boccara, A. Russo, P. Smith, and A. Gandjbakhche, "Enhancement of hidden structures of early skin fibrosis using polarization degree patterns and Pearson correlation analysis," J. Biomed. Opt. 10(5), 051706 (2005).

14. J. Falconet, R. Sablong, E. Perrin, F. Jaillon, and H. Saint-Jalmes, "Analysis of simulated and experimental backscattered images of turbid media in linearly polarized light: estimation of the anisotropy factor," Appl. Opt. 47(31), 5811-5820 (2008).

15. V. Backman, R. Gurjar, K. Badizadegan, I. Itzkan, R. R. Dasari, L. T. Perelman, and M. S. Feld, "Polarized light scattering spectroscopy for quantitative measurement of epithelial cellular structures in situ," IEEE J. Sel. Top. Quantum Electron. 5(4), 1019-1026 (1999).

16. M. Bartlett and H. Jiang, "Measurement of particle size distribution in multilayered skin phantoms using polarized light spectroscopy," Phys. Rev. E Stat. Nonlin. Soft Matter Phys. 65(3), 031906 (2002).

17. A. Sviridov, V. Chernomordik, M. Hassan, A. Russo, A. Eidsath, P. Smith, and A. H. Gandjbakhche, "Intensity profiles of linearly polarized light backscattered from skin and tissue-like phantoms," J. Biomed. Opt. 10(1), 014012 (2005). 
18. S. P. Morgan and I. M. Stockford, "Surface-reflection elimination in polarization imaging of superficial tissue," Opt. Lett. 28(2), 114-116 (2003).

19. S. L. Jacques, J. R. Roman, and K. Lee, "Imaging superficial tissues with polarized light," Lasers Surg. Med. 26(2), 119-129 (2000).

20. J. M. Schmitt, A. H. Gandjbakhche, and R. F. Bonner, "Use of polarized light to discriminate short-path photons in a multiply scattering medium," Appl. Opt. 31(30), 6535-6546 (1992).

21. S. P. Morgan and M. E. Ridgway, "Polarization properties of light backscattered from a two layer scattering medium," Opt. Express 7(12), 395-402 (2000).

22. L. Gobin, L. Blanchot, and H. Saint-Jalmes, "Integrating the digitized backscattered image to measure absorption and reduced-scattering coefficients in vivo," Appl. Opt. 38(19), 4217-4227 (1999).

23. H. J. van Staveren, C. J. M. Moes, J. van Marie, S. A. Prahl, and M. J. C. van Gemert, "Light scattering in Intralipid-10\% in the wavelength range of 400-1100 nm," Appl. Opt. 31(30), 4507-4514 (1991).

\section{Introduction}

2D optical imaging has proven a valuable tool for functional exploration in biological research such as in neuroscience (imaging the exposed cortex), ophthalmology (retinal imaging), or oncology (skin, uterus cancers detection and monitoring) [1-4]. Yet, there remains concern over the fact that images are two-dimensional (2D) projections of a combination of signals from superficial and deeper tissue layers, with an a priori unknown proportion. Conventional optical imaging of intrinsic signals (IOS) provides high-resolution functional 2D images, by monitoring changes in absorption of intrinsic chromophores. However, such systems do not allow depth discrimination. As in vivo IOS is usually performed under reflectance geometry [5], illumination sources and detectors belong to the same half-space. Under these conditions, these imaging systems suffer from (i) blurring due to mirror reflections at the interface; (ii) loss of depth information about the signals' origin, due to an indiscriminate collection of all photons, irrespective of their pathlengths. To tackle the first problem, one can use linear polarization illumination with cross-polarization detection, but in that case, the unpolarized backscattered detected photons are those who have undergone a large number of scattering events and hence a large proportion is likely to come from deep locations. Moreover, the selective detection of photons stemming from the surface or other specific depths remains a challenge.

The technique exposed hereafter is based on a polarimetric selection of the photons. Intuitively, when travelling through the medium, polarized photons will maintain their state of polarization to a different extent, depending on the absorption or scattering events, that is, according to the transport mean free path (MFP) [5-9]. The use of polarizing filters allows, up to a point, selecting photons for which polarization state is maintained: this is the basis of the polarization gating imaging methods. Linear polarization filters have been first extensively employed in sub-surface imaging, where one aims for surface mirror reflection elimination. A widely used technique is then detection via the cross-linear imaging channel to get rid of the mirror reflections. For tissues examination, this has initially been suggested by Anderson [10]. Applied to skin examination, these measurements are sensitive to depths superior to $300 \mu \mathrm{m}$ [11]. Surface contrast enhancement $[12,13]$ is the other main polarization gating application. A simple subtraction between collinear and cross-linear imaging channels allows the separation of the surface (or shallow subsurface) contribution from the multiply scattered deep volume part, resulting in an enhancement of the surface image. This has also been applied to the determination of the optical properties of biological tissues [14]. Polarization gating is also used for localized spectroscopy $[15,16]$ in order to extract surface signatures. Ellipsometry has also been performed on biological tissues: linear polarized illumination has been coupled with tunable polarization detection in order to characterize anisotropic structures in tissues, such as collagen fibers [17].

However, linear polarization gating, alone, does not allow filtering at the same time mirror reflections and multiple scattered light. In that sense, the technique proposed by Morgan and Stockford [18], advocating the use of circularly polarized light, is very appealing as it offers the possibility of filtering both mirror reflections and multiple scattered light, without any special preparation of the samples [11,19]. Since biological tissues are Mie scatterers [5], this filtration with circularly polarized light can indeed be achieved in tissues, allowing a deeper 
screening than linearly polarized light. MacKintosh et al. [6] introduced the concept of "polarization memory", describing the way the initial polarization is maintained through the variation of the number of scattering events, and showed that the polarization of circularly polarized light is indeed maintained through a larger number of scattering events than that of linearly polarized light. This effect has been demonstrated in biological tissues and used for the localization or analysis of specific sample regions not only in transmission image acquisition geometry [20] but also in reflection geometry [21], which is more adapted to biological tissue screening in vivo.

Here, we adopted the approach proposed by [18]. In addition, we introduce the novel idea that it is possible to select the probed depth as a function of the polarization ellipticity, thus allowing screening the tissue at any specific depth comprised between the surface and a maximum depth defined by the maximum penetration depth of the circularly polarized photons. An experimental setup has been developed and the principle is demonstrated through phantom experiments. Our final motivation in the present work is to test the feasibility of the technique for depth resolved IOI for exposed cortex in vivo examination. A first experiment has been conducted on an anesthetized rat and is reported as a first illustration.

\section{Materials and methods}

\subsection{Polarization gating for volume selection}

The principle has been developed by Morgan et al. [18]. It consists in sequentially illuminating the biological tissue with light polarized linearly or circularly. Detection performed under cross-linear polarization allows the collection of multiple scattered photons (those coming prevalently from deep tissues), whereas co-linear detection is restricted to the collection of linear polarization maintaining photons (mostly surface probing). If circularly polarized light is considered, one can distinguish three state of polarization domains: (i) the first backscattered photons have undergone a mirror reflection and thus flipped their helicity; when analyzed, those contribute to the cross-circular component (thus usable for the validation of the surface, mirror reflected, image); (ii) the second kind of backscattered photons emerge after a series of forward scattering events and maintain their original polarization state. They contribute to the co-circular component; (iii) the third type of photons have been randomly polarized by multiple scattering events and contribute equally to all polarization states.

By subtracting cross-linear images - to which the contribution of multiple scattered photons is predominant-from co-circular polarization images, one can probe a mediumdepth subsurface volume. The latter is free of mirror reflections, while eliminating the multiple scattered photons coming from greater depths (probed by the cross-linear image). The extent of this middle-depth, "subsurface" volume depends on the optical properties of the medium.

Importantly, the same reasoning as above can be applied to elliptical polarization. However, the probed volume will be shallower than for circular polarization.

\subsection{Experimental setup and image processing}

The experimental setup (Fig. 1) is composed of a 150W halogen light lamp (OSL1-EC, Thorlabs SAS, France) for illumination. The beam is homogenized through a telecentric objective, and wavelength filtered with an interference chromatic filter $(632.8 \mathrm{~nm}, 10 \mathrm{~nm}$ FWHW, Thorlabs SAS, France). The detector is a CCD camera (12 bits, Orca ER, Hamamatsu Photonics, Japan) with an objective (7000E, Laser Components SAS, France) for detection. The incident beam passes through a polarizer and quarter wave plate. In the detection path there are also a quarter wave plate and an analyzer. The relative rotation angle between polarizers and quarter wave plates is tuned such as to obtain the desired polarization modes for illumination and detection.

The axes of the different optical components were first set with calibrated measurements. Linear polarization is obtained, for a given polarizer/quarter-wave plate couple, when the axis 

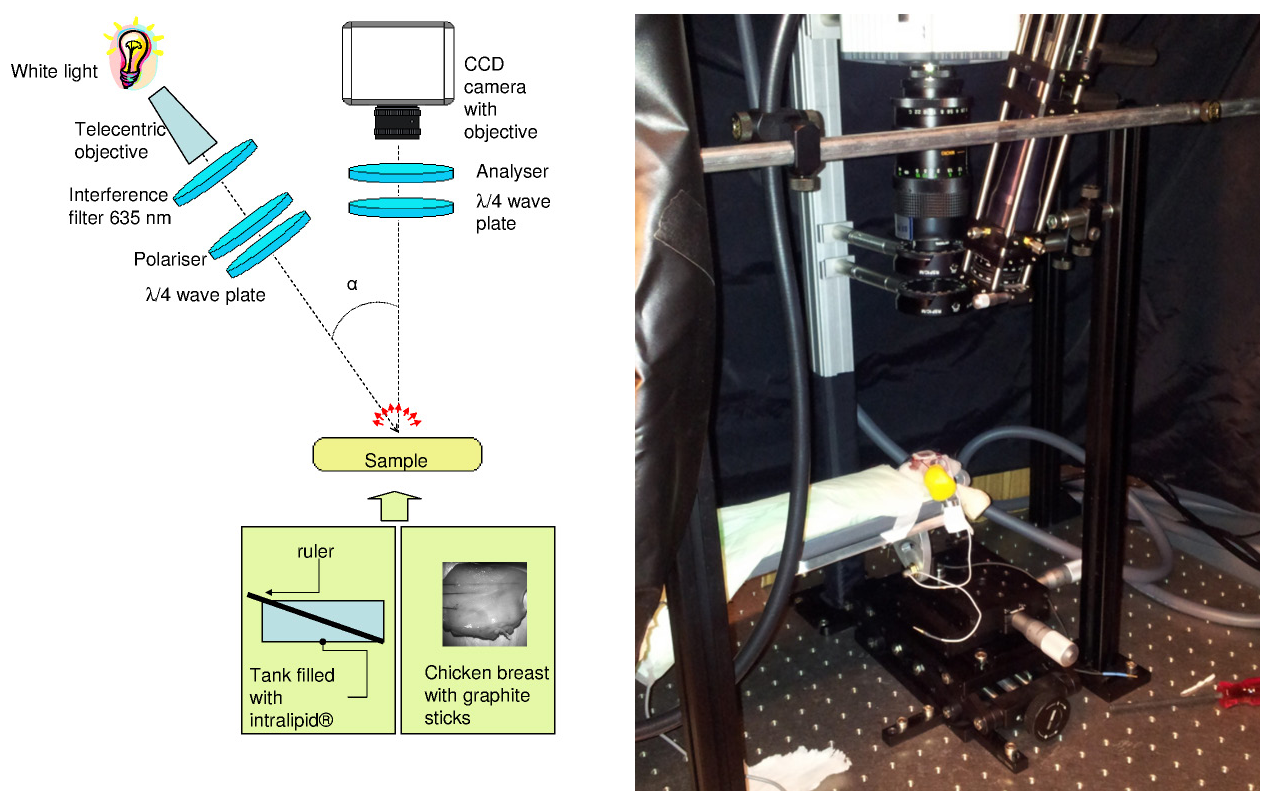

Fig. 1. Sketch (left) and photograph (right) of the experimental setup.

of the polarizer and the quarter-wave plate are aligned; circularly (right or left) polarized light is obtained when the fast axis of the quarter-wave plate is $\theta=45^{\circ}$ ( + or - , respectively) off the linear polarizer axis.

Experiments have been performed under different illumination/detection channels: (i) "collinear" image (illumination under linear polarization and detection under co-linear polarization) detects surface mirror reflected signal plus volume scattered signal; (ii) "crosslinear" image (illumination under linear polarization and detection under cross-linear polarization) detects only volume scattered signal; (iii) "co-elliptical" images (illumination under elliptical polarization and detection under co-elliptical polarization) detect subsurface signal and volume scattered signal.

With these schemes, the following imaging channels can be defined as follows:
(A) "surface" = "collinear" - "crosslinear";
(B) "middle" = "co-elliptical" - "crosslinear";
(C) "multiple scattered" = "crosslinear".

Evaluation of the polarization-maintained signal from the middle channels is based on the assumption that the fraction and characteristics of the multiple scattered photons are the same for linearly and elliptically polarized light. This approximation is substantiated in [19] for the case of circularly polarized light. In this reference, a Monte Carlo simulation shows that the penetration depth profiles of the collected multiple scattered photons are the same under cross-circular and cross-linear configurations, even if the number of multiple scattered photons collected under cross-circular configuration is smaller than the number collected under cross-linear configuration, which is coherent with the above-mentioned "polarization memory" effect. The image resulting from the subtraction of the middle channel and the multiple scattered channel, approximated by the measurement under cross-linear configuration, contains indeed only polarization-maintained signal, but this signal is underestimated, leading to images with a poorer signal to noise ratio (SNR). Although the reasons of the similarity of the depth distributions of multiple scattered photons, collected under both polarization configurations, are not obvious, the same approximation has been adopted here for light polarized elliptically. A numerical verification (Monte Carlo 
simulations not shown here) has shown indeed this approximation has no major impact on the resulting images, provided, at least, the anisotropy factor is high, which is the case in biological tissues. The validity of this approximation depends indeed on the optical properties of the medium and deserves a specific study, which is out of the focus of this paper.

The exposure time of the camera was adapted to each measurement in order to preserve a high SNR for each measurement. Yet, to facilitate comparison between different images, the data are expressed per unit exposure time. No other processing has been applied to the images.

\subsection{Samples description}

Experiments have been conducted on three kinds of samples: the first series on Intralipid liquid phantoms for quantification evaluation, the second on a piece of chicken breast for biological tissue feasibility, and the third one, on an anesthetized rat with exposed cortex, as in vivo test.

\subsubsection{Liquid phantom}

The liquid phantom was composed of aqueous Intralipid (20\%, Sigma-Aldrich, France) dilution at adequate concentrations. A target in the form of a ruler (plastic) had been placed obliquely in a tank according to the sketch in Fig. 1. Placed in that way, each graduation read on the CCD screen corresponded to a different depth. The concentration of Intralipid had been adapted to match biological tissues optical properties but with a scaling in the size of the sample for macroscopic measurement of depth. The optical properties of Intralipid $1 \%$ have been determined according to the integral reflectance method [22]: the absorption coefficient is negligible and a $\mu_{s l \%}^{\prime}=10.3 \pm 0.5 \mathrm{~cm}^{-1}$ reduced scattering coefficient value was estimated. According to the accuracy of our optical (magnification) and mechanical components, a $0.1 \%$ diluted solution had been adopted, corresponding to $\mu_{s 0.1 \%}^{\prime}=0.95 \pm 0.5 \mathrm{~cm}^{-1}$, allowing depth measurements at a millimetric scale. The anisotropy factor of Intralipid has been estimated to $g=0.73$ [23] leading to a scattering mean free path $M F P=(1-g) / \mu_{s}^{\prime} \sim 2.8 \mathrm{~mm}$ (that is approximately 10 times longer than in biological tissues).

\subsubsection{Biological phantom}

The second phantom was a piece of chicken breast. A graphite stick (diameter, ca. $1 \mathrm{~mm}$ ) was inserted with a small tilt angle (exact value not evaluable) to serve as inclusion. Caution was taken though to keep the superficial tissue sufficiently hydrated. This helped tracking the mirror reflections.

\subsubsection{In vivo experiment}

After initial anaesthesia with $5 \%$ isofluorane, an adult male Wistar-Han rat was anesthetized with Urethane (intraperithoneal injection, $130 \mathrm{mg} / 100 \mathrm{~g}$ body weight) placed in a stereotaxic frame for mechanical stability during craniotomy and during all the duration of the recordings. Body temperature was monitored, and maintained between 36 and $38^{\circ} \mathrm{C}$ with the use of a warm water bag. A craniotomy was performed creating a cranial window of ca. $5 \mathrm{mmx} 5 \mathrm{~mm}$ above the barrel cortex. To prevent the cortex from drying, a chamber of dental cement was created around the entire cranial opening such as to create a chamber that was filled with agarose in saline $(2 \%)$. To provide a flat optical interface, the chamber was sealed with a microscope coverslip. In compliance with the directions of the Local Ethics Committee, we kept the number of animals used for this study as small as possible. All procedures were in agreement with NIH Guidelines.

\section{Results}

The two phantoms described above have been examined under the various described imaging channels. Hereafter, we show the resulting images obtained for the different "middle" channels and the "volume" channel. 
The results obtained in Intralipid $0.1 \%$ are represented on Fig. 2. In the top part of the image, the different images are displayed with the same colorbar and show that the ruler can be seen in progressively larger depth, with a signal whose amplitude increases as well, showing that an increasing number of photons are collected as the polarization ellipticity increases. The middle part of Fig. 2 displays images with scales adapted (automatic scaling between maximum and minimum, with jet color map) to each image in order to enhance the contrasts of low signal images (subsurface images). It shows explicitly that some deep

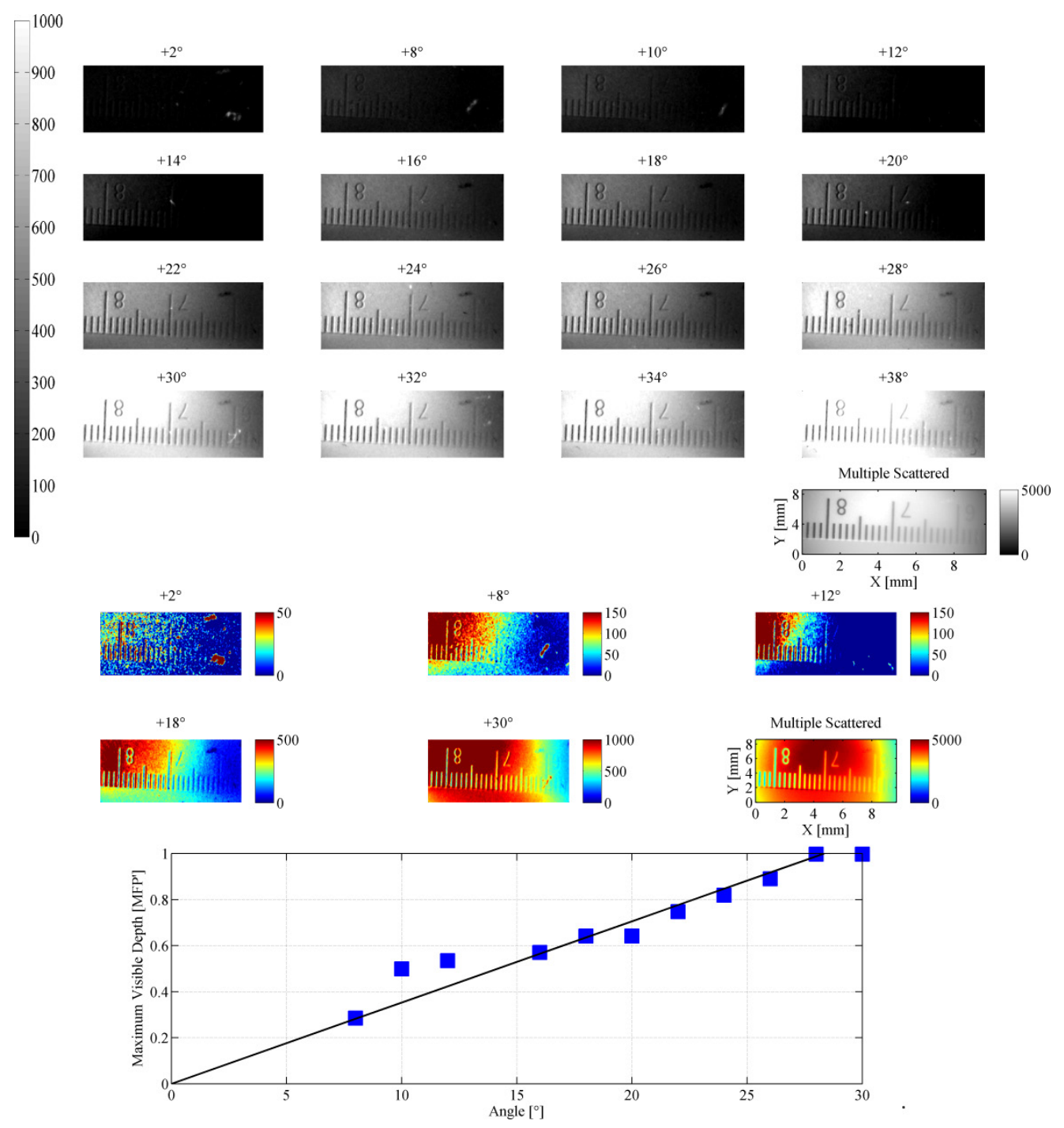

Fig. 2. Top: four first rows, "middle" channels images, obtained at different degrees of ellipticity, for Intralipid $0.1 \%$ experiment. The angle value measures the angle $\theta$ between the polarizer and the illumination quarter-wave plate. The same grey level scale was used for all images. Fifth row image, deep channel image obtained under crosslinear detection, with the same field of view but different grey level scale (see colorbar). $\mathrm{X}$ axis indicates the actual depth of the ruler in the Intralipid, increasing from left to right, while $Y$ axis indicates the actual dimension read at the surface of the probed medium. Middle: selected images with color level scale adjusted to each image, such as to go from its minimum to its maximum. For all images, intensity is expressed in arbitrary units. Bottom: maximum depth (expressed in MFP' $\left.=\left(\mu_{s}^{\prime}\right)^{-1}, 1 \mathrm{MFP}^{\prime}=1.05 \mathrm{~cm}\right)$ visible in a given polarization maintained channel as a function of the corresponding angle $\theta$ between the polarizer and the illumination quarter-wave plate, the fitting line y $=0.035 \mathrm{x}$ (linear regression coefficient $R^{2}=0.89$ ) is represented (black line). 
structures are not visible on subsurface channels while clearly visible on deeper channels: for instance, number " 7 " is not visible on channel $\theta=$ " $+8^{\circ}$ " while clearly visible on channel $\theta=$ " +18 o".

Calculated from the tilt angle of the ruler, here, $1 \mathrm{~mm}$ read on the image corresponds to $0.375 \mathrm{~mm}$ depth shift: the horizontal axis on the multiple scattered image represents the corresponding actual depth of the ruler in the Intralipid, while the vertical axis indicates the actual dimension read at the surface of the probed medium. This experiment shows that polarized light can be collected up to $\sim 5 \mathrm{~mm}$ in depth (corresponding to $0.5 \mathrm{~mm}$ in biological tissues) without any difficulty. To fix the ideas, the depth of the last visible bar of the ruler (Maximum visible depth), read in a given polarization maintained channel, is plotted as a function of the corresponding angle $\theta$. In order to have an estimation of the depth accessible in tissues, this depth is reported with a MFP' (transport mean free path) scale, accounting for all optical properties. A linear behavior is found showing a complete correlation between the polarization ellipticity and the probed depth, up to $\theta=$ " $+30^{\circ}$ ". This experiment can be easily reproduced for different optical properties media (for different absorption, scattering coefficients and anisotropy factors) and could serve as calibration measurement for tissue studies.

As the result of images subtraction, the SNR of the resulting channel images is smaller than that of the measured images. Using basic image processing should allow to reduce the noise contain of the images. Interestingly, a specific statistical analysis of the images as the Pearson correlation analysis proposed in [13] could improve the accuracy of the estimation of the probed depth.

The chicken breast experiment (Fig. 3) illustrates the feasibility in other kind of tissues. The graphite stick had been inserted from left to right with a small angle such that its depth is higher on the right hand side of the image. The horizontal and vertical axes, reported only on the multiple scattered images, indicate the field of view at the surface of the probed medium, in pixels unit, 1 pixel $=39 \mu \mathrm{m} \times 39 \mu \mathrm{m}$. The depth of the tip of the stick has not been properly measured: the coefficient of proportionality between the horizontal axis and the actual depth is not reported. However, we reported on the bottom part of Fig. 3 the maximal position (pixel units) of the visible part of the graphite stick, read on the horizontal axis of images in Fig. 3, as a function of the angle $\theta$. Basically the same comments as above could be done, small angle $\theta$ representing near surface images, that is small polarization ellipticities, show only the superficial part of the stick (left side of the image) while for larger angle $\theta$, that is higher polarization ellipticities, right side becomes more and more visible, with an increase of the signal intensity.

The in vivo results are reported on Fig. 4. A network of blood vessels having different sizes can be seen on the white light image (Fig. 4, Top left). We focused on a portion of the image (Fig. 4, Top right) showing two distinct absorbing structures of different sizes: a very large vessel (a), and a smaller one (b). The first one (a) is a large superficial structure and is present in all channels. The smaller vessel (b) is visible only beyond a certain degree of ellipticity $\left(\theta \geq+14^{\circ}\right)$. Moreover, the signal intensity increases more above vessel (a) than below it. This discrepancy is not visible in the deep channel image, suggesting that the observed local absorption pattern is restricted to a more superficial volume.

\section{Conclusion}

Here, we reported on the usage of polarization gating imaging with elliptically polarized light. Experiments were conducted both on phantoms and in vivo. Our results show that using imaging channels with elliptically polarized light allows probing subsurface volumes, which are well defined. Whereas the exact depth and extent of these subsurface volumes depend on the optical properties of the medium, such polarization gating allows producing images free of surface and deep volume scattering contaminations. Our phantom experiments show that the method can be used to probe tissues in depth up to at least $0.5 \mathrm{~mm}$, which offers the possibility of screening a variety of layered biological tissues such as the exposed cortex, as tested here, or the skin. Further work, including modelling of light propagation, improvement

\#170638 - \$15.00 USD

(C) 2012 OSA 
of the setup and application of signal processing, should allow using the method to image selectively sub-volumes at well-defined, specific depths. In this sense, the presented approach constitutes a first step towards functional optical imaging at user-selected depths within the tissue.

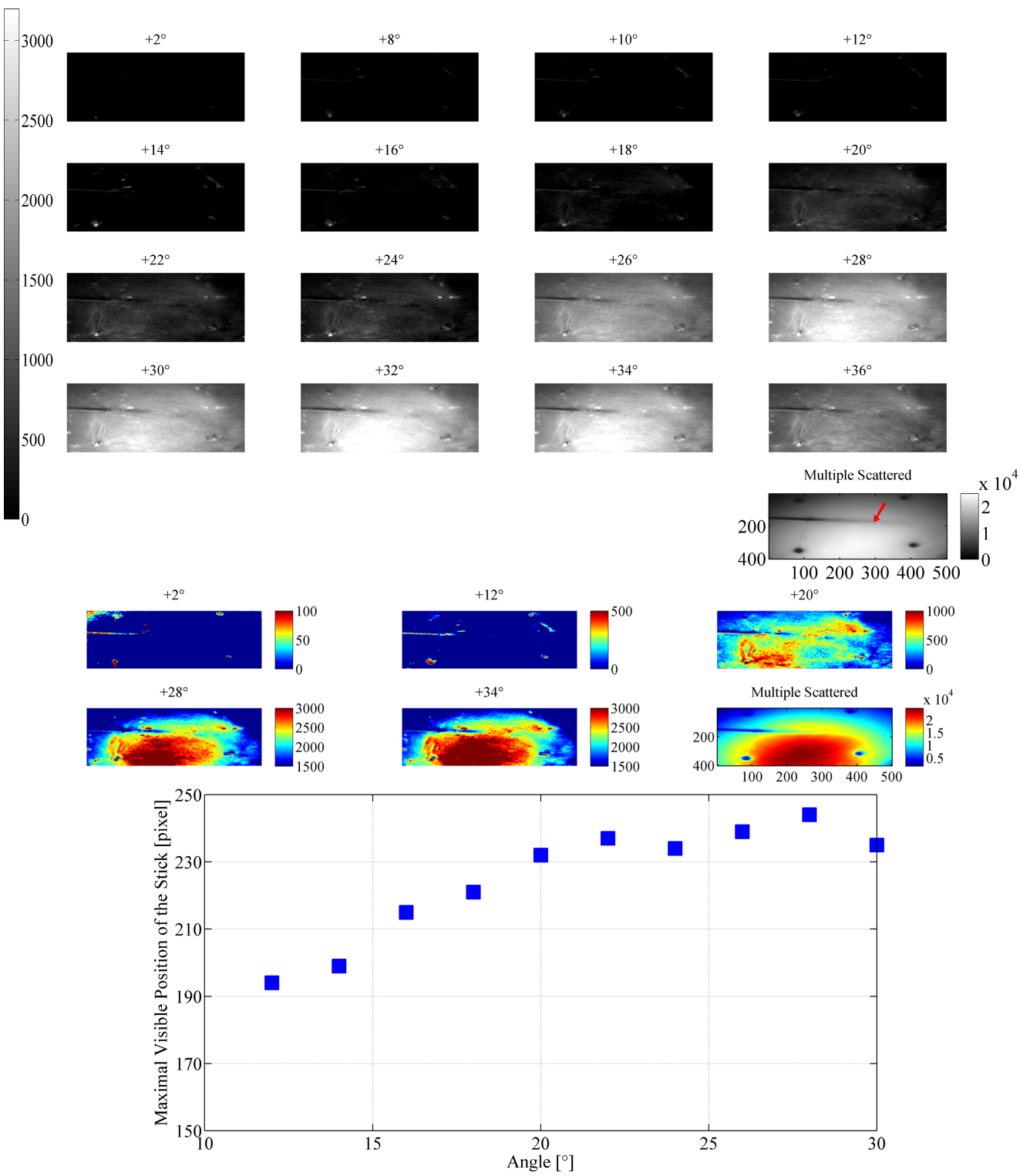

Fig. 3. Same as in Fig. 2 for chicken breast experiment. The depth of a graphite stick (arrow) in the tissue increases from left to right. Horizontal and vertical axes of images represent the field of view. Pixel size: $39 \mu \mathrm{m}$. 

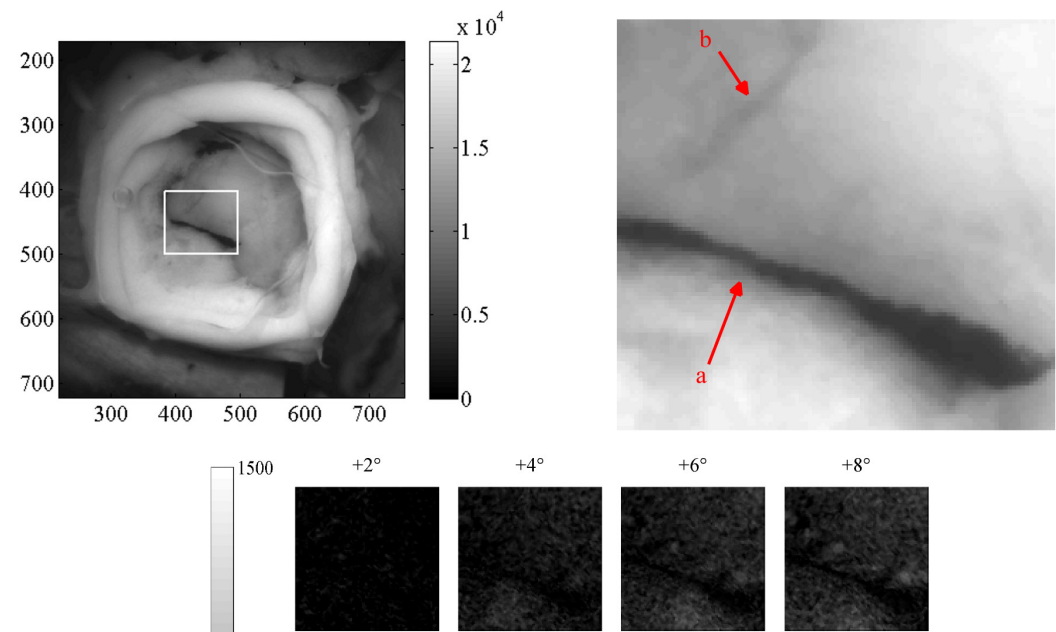

$+6^{\circ}$

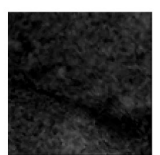

$+8$

$+10^{\circ}$

$+12^{\circ}$

$+14^{\circ}$

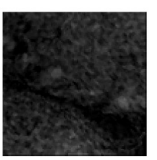

$+16^{\circ}$

$-1000$

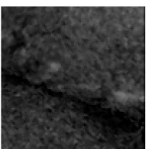

$+18^{\circ}$
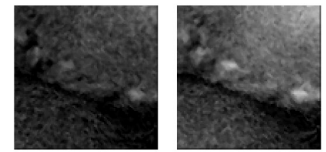

$+22^{\circ}$

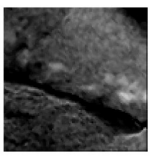

$+24^{\circ}$

500

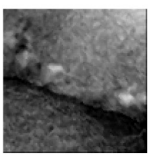

$+20^{\circ}$
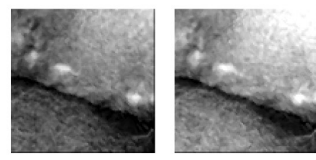

$+26^{\circ}$

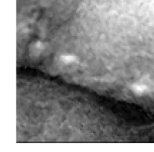

$+28^{\circ}$

$+30^{\circ}$

Multiple Scattered
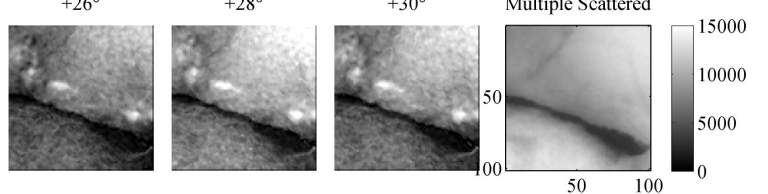

$+2^{\circ}$

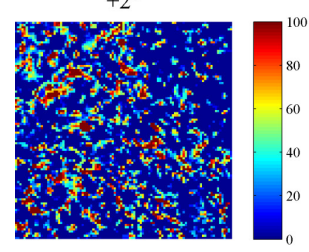

$+6^{\circ}$
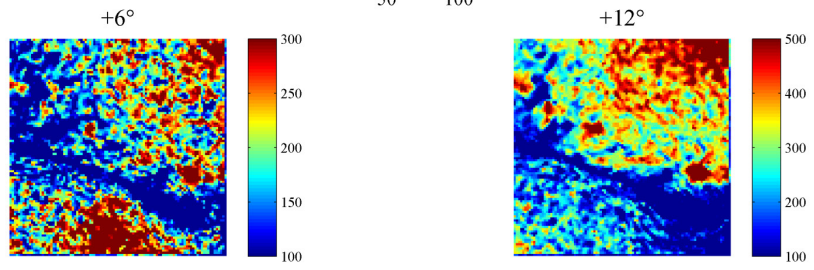

$+30^{\circ}$
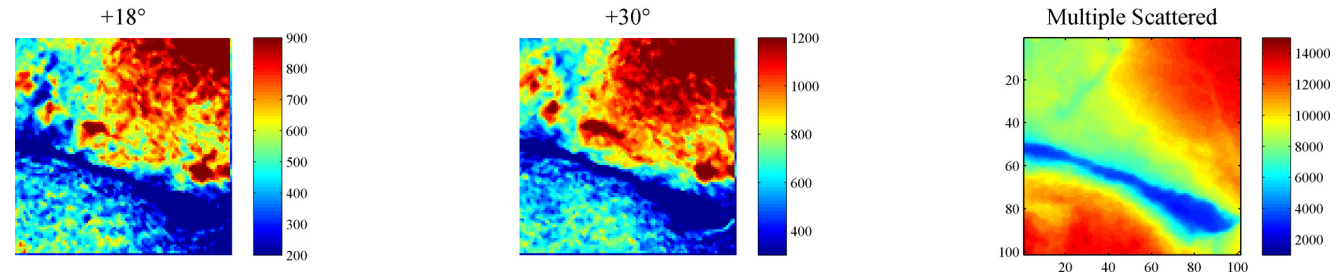

Fig. 4. Top, white light image of the imaging window with a large field of view (left), white rectangle marks the reduced field of view in which the image processing is performed (right). Middle and bottom, same as in Fig. 2, for rat cortex examination. Pixel size: $42 \mu \mathrm{m}$.

\section{Acknowledgments}

We thank Dr. N. Montejo Cervera for help with the rat experiment. Part of this work was supported by a grant from NRJ and recurrent CNRS funding.

\#170638 - \$15.00 USD

(C) 2012 OSA
Received 18 Jun 2012; revised 2 Aug 2012; accepted 2 Aug 2012; published 23 Oct 2012

1 November 2012 / Vol. 3, No. 11 / BIOMEDICAL OPTICS EXPRESS 2915 